\title{
Dynamic interferometric measurement with extended unambiguity range in flow measurement
}

\author{
Pavel Psota ${ }^{1 *}$, Roman Doleček ${ }^{1}$, Vit Lédl $^{1}$, and Tomáš Vít ${ }^{2}$ \\ ${ }^{1}$ Institute of New Technologies and Applied Informatics (NTI), Faculty of Mechatronics, Informatics and Interdisciplinary Studies, \\ Technical University of Liberec, Studentská 2, 46117 Liberec, Czech Republic \\ ${ }^{2}$ Institute of Energetic Devices (KEZ), Faculty of Mechanical Engineering, Technical University of Liberec, Studentská 2, 46117 \\ Liberec, Czech Republic
}

\begin{abstract}
This paper reports on a new approach to measure dynamic processes in fluid mechanics using interferometry with extended dynamic range. A key factor is the use of two wavelengths and the recording of interferograms from both wavelengths in one frame. Phase map evaluation is based on the Fourier transform. The difference between the obtained phase fields creates a synthetic phase whose dynamic range also covers large changes of the measured quantity.
\end{abstract}

\section{Introduction}

Interferometric methods such as digital holographic interferometry and interferometry are generally understood to belong to the group of most accurate measuring devices [1]. Their fundamental measure is the wavelength of light and the todays interferometer can resolve even a fraction of the wavelength, so their sensitivity is outstanding. Due to their great accuracy interferometers in different variations are used in many branches of measurement including the fluid mechanics. However, due to the bounded nature of a light wave, the measurement range given by the unambiguity is squeezed within the range of one cycle of the wave. Measured phase maps are therefore wrapped within the range of $2 \pi$ radians.

Unless the measured temperature field shows a small gradient (there is no violation of the Nyquist conditions when sampling the interferogram by the camera), the phase field can be expanded using standard 2D algorithms to obtain a smooth phase field without jumps. The ambiguity of interferometric measurement requires knowledge of phase change at least at one point. If we consider the phenomenon taking place only in a small area in the middle part of the field of view (e.g. jets), it can be assumed that the change at the edge of the field of view will be minimal and does not cause a phase change of more than $2 \pi$. This area can be considered as a reference wave and can be used as a starting position for unwrapping procedure. The measurement is then absolute in terms of temperature change.

However, many tasks in the flow area do not have these properties. Often there is a significant change in the measured quantity across the whole field of view. As an example, you can measure heat transfer inside the prime mover resonator or flow around the wing at high speeds. If we want to quantify the observed phenomenon correctly, we must measure this quantity at least at one point by another method. For instance, if we measure the temperature gradient inside the cavity, a single-point temperature probe can be placed here. The value at this point will be the reference value at which the measured values can be related to the interferometric value. This approach is naturally in contradiction with the big advantage of contactlessness of interferometric measurement.

Multiple wavelengths [2] interferometry can be used to increase the dynamic range of the measurement. Two or more interferograms at different wavelength are successively captured and the phase information is combined in order to get higher dynamic range of the measurement. These measurement methods are suitable for stationary phenomena that are quite rare in the fluid mechanics measurement. In this paper we propose a method based on carrier frequency interferometry with simultaneous use of two wavelengths. This makes it possible to vary the dynamic range of measurement almost arbitrarily and it is therefore possible to measure in real time phenomena causing large phase changes without the need for a reference gauge.

\section{Principle of the method}

Digital interferometry is a very accurate measurement method that is sensitive to the change of optical wave phase. By recombining two coherent waves, an interference pattern $I$ is created in Cartesian coordinates $(x, y)$ described by the equation:

$$
I(x, y)=A(x, y)+B(x, y) \cos (\varphi(x, y))
$$


where A represents additional component, B is multiplicative component and $\varphi$ stands for phase. Phase-shifting interferometry (PSI) is usually used for quantitative phase evaluation of the phase map $\varphi(x, y)$. In PSI the phase shift is repeatedly added to the cosine function argument of equation (1). There are many ways how to generate the phase shift in interferometers like a slight movement of mirror in reference arm or wavelength change. From (1) follows that at least 3 interferograms must be recorded (three equations for three variables $\mathrm{A}, \mathrm{B}$, and $\varphi$ ), and therefore PSI is not suitable for measuring of dynamic events.

In contrast, the spatial carrier frequency interferometry $(\mathrm{SCI})$ introduces carrier frequencies $v_{x}$, $v_{y}$ into a well-known interference equation (1):

$$
I(x, y)=A(x, y)+B(x, y) \cos \left(2 \pi v_{x} x+2 \pi v_{y} y+\varphi(x, y)\right)
$$

This generates a high density interference pattern. Due to the carrier frequencies, we are able to separate the wavefront, its conjugate part and the DC term, see Fig.1. After performing the FT, we get the phase map directly as an angle of the complex field. SCI is therefore suitable for measurement of fast phenomena since the phase is evaluated from single hologram. For proper working of SCI it is necessary to set the carrier frequencies correctly so that all the spectral parts can be separated and in the same time the sampling criterion (Nyquist frequency $v_{N}$ ), which is given by the pixel extension of the digital image sensor, is not violated. In practice one can observe the Fourier spectra of the interferogram in real time and shift the wavefront in e.g. $v_{x}=v_{y}=0.5 v_{N}$ by adjusting the tip/tilt of a mirror in the reference arm of the interferometer, see Fig. 1c.
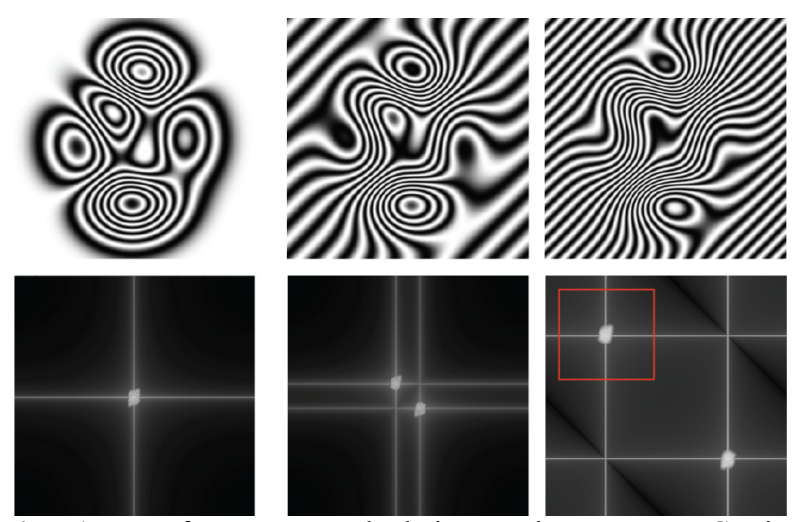

Fig. 1. Interferograms and their Fourier spectra. Carrier frequencies are set to a) $v_{x}=v_{y}=0$; b) $v_{x}=v_{y}=0.1 v_{N}$; c) $v_{x}=v_{y}=0.5 v_{N}$. The red rectangle denotes spectral part of the wavefront used for the phase retrieval.

Graphically the process of processing is described in Fig. 2. The output is a wrapped phase map that is in the interval $[-\pi, \pi]$. This limitation causes ambiguity in interferometric measurement as described in the introduction chapter. Particularly, the phenomenon can generate maximal phase change to be $\pi$. a)

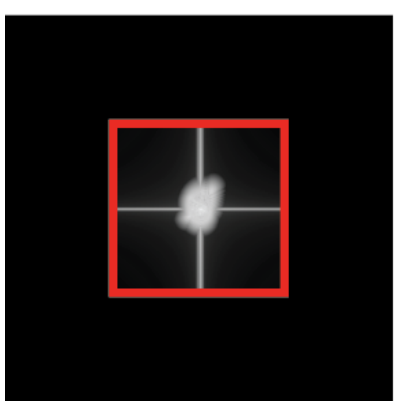

b)

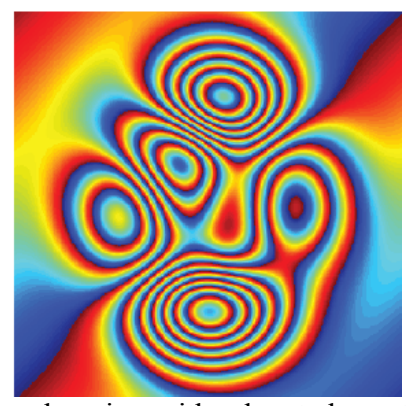

Fig. 2. A) Filtered Fourier domain with denoted part corresponding to Fig. 1c and b) wrapped phase obtained as an angle of the complex field computed by inverse Fourier transform of spectrum shown in a).

Multi-wavelength interferometry allows for extension of the measurement range. If the interferometer employs two wavelengths $\lambda_{1}, \lambda_{2}$, the difference between the phases $\varphi_{1}, \varphi_{2}$ is called synthetic phase $\Phi$ :

$$
\Phi=\varphi_{2}-\varphi_{1}=\frac{4 \pi}{\Lambda} z
$$

that corresponds to the phase with the synthetic wavelength:

$$
\Lambda=\frac{1}{1 / \lambda_{1}-1 / \lambda_{2}} .
$$

The synthetic wavelength is larger than the optical wavelengths $\lambda_{1}, \lambda_{2}$ and determines the range of unambiguousness to $\Lambda / 2$. The synthetic phase therefore provides greater measurement range. On the other hand, the uncertainty in interferometric measurement is in fraction of the used wavelength. Therefore the synthetic phase $\Phi$ suffers from noise more than the individual phases $\varphi_{1}, \varphi_{2}$.

In the case of dynamic measurement, the condition of recording and evaluation from one interferogram remains. It is therefore not possible to first record one interferogram with use of the first wavelength and then repeat the measurement with the second wavelength as the measured variable changes over time.

However, formula (2) and Fig. 1 show that the magnitude carrier frequencies and their directions determine the position of the wavefront in the spectral domain. Taking advantage of this property and considering interferometry with two different wavelengths at the same time on the camera chip, we can achieve their spectral separation and reconstruct the phase fields without affecting them. The interference equation (2) is then extended by another interference member yielding in: 


$$
\begin{aligned}
& I(x, y)=A(x, y)+ \\
& +B_{1}(x, y) \cos \left(\omega_{x 1} x+\omega_{y 1} y+\varphi_{1}(x, y)\right)+ \\
& +B_{2}(x, y) \cos \left(\omega_{x 2} x+\omega_{y 2} y+\varphi_{2}(x, y)\right)
\end{aligned}
$$

Same subscripts correspond to the same wavelengths. By adjusting the carrier frequencies for the different wavelengths in the perpendicular directions, spectral separation of the phase information can be achieved.

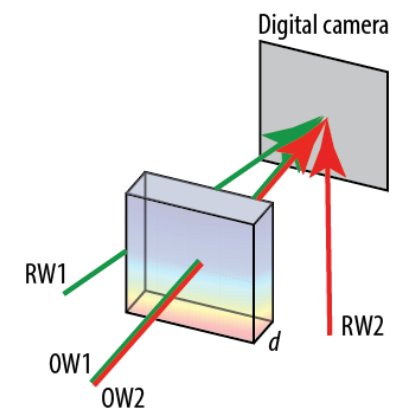

Fig. 3. Principle scheme of DWSCI. Objet waves (OW1, OW2) integrating the measured phase change imping perpendicularly on the CCD or CMOS while the reference waves for different wavelengths (RW1, RW2) imping tha sensor at different angles in order to generate different spatial carrier frequencies.

The phases at the both wavelengths are then retrieved in the same manner as described in the previous section and the synthetic phase can be computed (3). The synthetic phase has an increased range but for the price of lower accuracy. Therefore, the synthetic phase is suitable as a good estimate and one can use its values in order to correct the phase map obtained from single wavelength measurement. This approach yields in dual wavelength spatial carrier interferometry (DWSCI). This enables high dynamic range measurement and high accuracy in real-time measurement. For the sake of clarity, it is necessary to note that the recording depends on the frame rate of the camera (can be high speed camera with frame rate in $\mathrm{kHz}$ ), and the evaluation is then given by the computing time on the computer used (in order of few seconds for common PC). However, the evaluation can also be done off-line after the experiment ends.
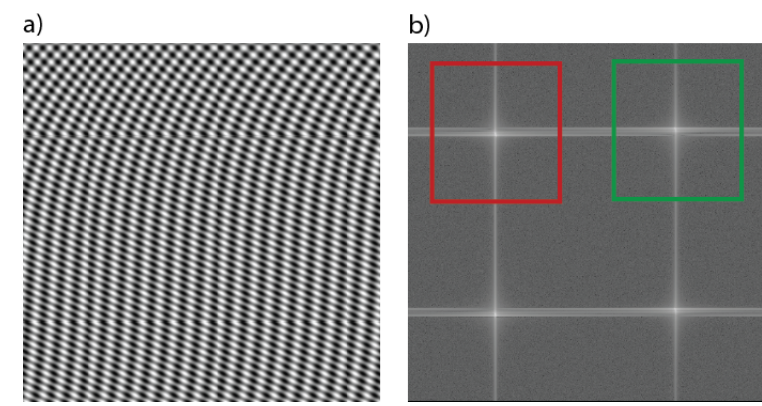

Fig. 4. a) Interferogram obtained by DWSCI and b) its Fourier sepctrum. Red and green rectangles represent wavefronts of generated by the measured phenomena at different wavelengths.

\section{DWSCI - case study}

This section will introduce simulations result showing basic features and benefits of DWSCI.

Let us consider a 2D temperature field (the temperature values along $\mathrm{Z}$-axis are constant e.g. temperature field in a cavity) [3] having length $d=10 e^{-2} \mathrm{~m}$ and the initial temperature $25^{\circ} \mathrm{C}$. In the measurement time the temperature field has changed and the temperature change map is introduced in Fig.5a. The maximum temperature change is cca $290^{\circ} \mathrm{C}$ and the minimum is $30^{\circ} \mathrm{C}$. The temperature field is related to the refractive index $n$ by formula [4]:

$$
T=\frac{\frac{A}{n-1}-1}{B},
$$

where $\mathrm{A}=0.000294036$ and $\mathrm{B}=0.00368184$. And finally the interferometers are sensitive to phase $\varphi$ change caused by the variation in refractive index $\Delta n$ :

$$
\varphi=\frac{2 \pi}{\lambda} \Delta n d
$$

The typically used coherent source in interferometry is a double-frequency Nd:YAG laser having wavelength of $532 \mathrm{~nm}$. The phase change caused by the heating of the space in the field of view (Fig. 5 a) is shown in Fig. 5 b.
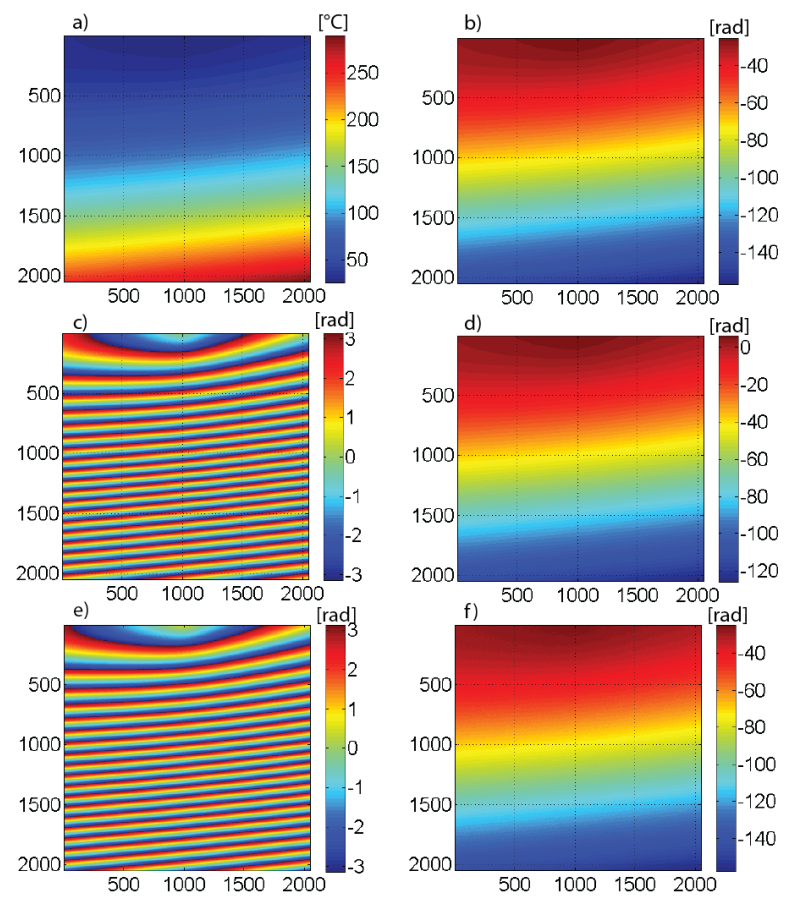

Fig. 5. a) Simulated temperature change; b) absolute phase corresponding to temperature change in a); c) wrapped phase as measured by single wavelength interferometry at $532 \mathrm{~nm}$; d) unwrapped phase map from c) with wrong scale due to ambiguity of interferometric measurement; e) wrapped phase as measured by single wavelength interferometry at $514.5 \mathrm{~nm}$; f) resulting phase map computed from synthetic phase and providing scale in agreement with $b$ ). 
From the computed (ideal) phase field we generated the interference pattern and processed the data in order to get phase information again. Due to the ambiguity of the intererometric measurement, we obtained measured wrapped phase field regardless of the way of evaluation (PSI, SCI, ...). The wrapped phase map is shown in Fig. 5c. After application of phase unwrapping algorithm, we obtain a smooth (without $2 \pi$ jumps) phase field, see Fig. 5d. Qualitatively the two phase fields (ideal and measured) are in agreement; however, their absolute value varies and therefore the absolute temperature distribution cannot be measured accurately.

Therefore, another approach, such as DWSCI, must be applied. Let us add a second light source - e.g. Ar laser - with wavelength 514.5nm to the experiment. By setting the correct carrier frequencies (the incident angle of the reference waves on the sensor), we get the interferogram as shown in Fig. 4a described by

$$
\begin{aligned}
& I(x, y)=A(x, y)+ \\
& +B_{1}(x, y) \cos \left(0.5 \omega_{N} x+0.5 \omega_{N} y+\varphi_{1}(x, y)\right)+. \\
& +B_{2}(x, y) \cos \left(-0.5 \omega_{N} x+0.5 \omega_{N} y+\varphi_{2}(x, y)\right)
\end{aligned}
$$

The frequency spectrum of the interferogram is shown in Fig. 4b. It is clearly seen the spectral separation of the both spectral components of the captured wavefronts marked with rectangles. Different colors denote different wavelengths. The phase fields are retrieved as mentioned above by filtering out the carrier frequency in Fourier domain followed by inverse Fourier transform. Reconstructed wrapped phase fields are shown in Fig, 5e, respectively. From the both phase maps, a synthetic phase can be computed (3). Using formula (4) synthetic wavelength yields in $15.6 \mu \mathrm{m}$ and provides sufficient measurement range.

As the noise in the phase field is directly proportional to the wavelength, the synthetic phase map suffers much more with noise than the phase field computed from single wavelength measurement. Several procedures can be used to refine the synthetic phase map in order to gain advantage from the both approaches: large measurement unambiguity of synthetic phase and accurate measurement of single wavelength phase.

The easiest and most straightforward procedure is to use the synthetic phase to detect the average phase shift (in terms of fringe order $\mathrm{N}$ ) across the field of view by:

$$
N=\operatorname{round}\left(\frac{\left\langle\Phi(x, y) \frac{\Lambda}{\lambda_{2}}\right\rangle-\left\langle\varphi_{2}(x, y)\right\rangle}{2 \pi}\right),
$$

where \langle\rangle operator stands for average. In described example the fringe order is $N=-5$. The unwrapped phase $\varphi_{2}$ providing relative information can be adjusted in order to have absolute measurement by

$$
\varphi_{2}^{A B S}(x, y)=\varphi_{2}(x, y)+2 \pi N .
$$

Fig. 5f shows the phase field obtained by (10) that is in agreement with ideal phase map and thus the method provides absolute interferometric measurement.

Other procedures, which can be used for DWSCI are performed independently in each pixel. This is for example a hierarchical phase unwrapping [5] or a robust retrieval introduced by authors in [6]. All approaches will theoretically finish with the same results; however, in the case of disturbing signals, it is more appropriate to use a robust evaluation that gives reliable results even in the presence of, for example, dispersion caused by a large wavelength difference.

\section{Conclusions}

This article introduces a new approach to measure dynamic processes in fluid mechanics using interferometry with extended dynamic range. A key factor is the use of two wavelengths and the recording of interferograms from both wavelengths in one frame. Phase map evaluation is based on the Fourier transform, and therefore the spectral separation of the phase information from both wavelengths can be achieved when the hardware of the experimental arrangement (angles of the reference waves) is correctly set. The difference between the obtained phase fields creates a synthetic phase whose dynamic range also covers large changes of the measured quantity. However, the synthetic phase is more influenced by noise. By combining the synthetic phase and the phase obtained from single wavelength, measurement can be made more precise to the value of standard interferometric technique. In conclusion, DWSCI has the same accuracy as SCI but with a significantly higher range of measurement.

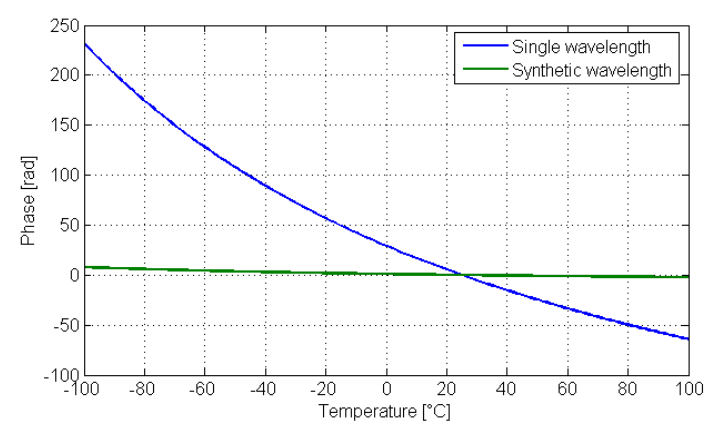

Fig. 6. Dependence of phase change on temperature change. As the temperature varies from its initial value $\mathrm{T} 0=25^{\circ} \mathrm{C}$ the phase of the object wave changes. Phase ambiguity range $[-\pi, \pi]$ in case of single wavelength is reached for small temperature change while the synthetic phase allows for much larger temperature changes.

Fig. 6 shows how the measurement range can be extended. Considering the same parameters as in previous section, the maximal change in temperature of SCI (being inside ambiguity interval $[-\pi, \pi]$ ) is approximately $25^{\circ} \mathrm{C} \pm 3^{\circ} \mathrm{C}$. In case of DWSCI (green line) the interval is approximately $[-41,146]^{\circ} \mathrm{C}$. 


\section{Acknowledgments}

We gratefully acknowledge the support of the Grant Agency of the Czech Republic - Czech Science Foundation (projects no. 16-16596S).

\section{References}

1. D. Malacara, Optical Shop Testing. 2007.

2. K. Meiners-Hagen, R. Schödel, F. Pollinger, A. Abou-Zeid, MEASUREMENT SCIENCE REVIEW, 9 (2009).

3. R. Doleček, P. Psota, V. Lédl, T. Vít, J. Václavík, and V. Kopecký, Applied Optics, 52, 1, (2013).

4. C. M. Vest. Holographic Interferometry. 1979.

5. Ch. Wagner, W. Osten, S. Seebacher, Opt. Eng., 39, 1, (2000).

6. P. Psota, V. Lédl, P. Vojtíšek, O. Matoušek, Proc. SPIE 10151, 1015113, (2016). 